\title{
MIGRACIÓN INTERNACIONAL Y SALUD: EL APORTE DE LAS TEORIAS SOCIALES MIGRATORIAS A LAS DECISIONES EN SALUD PÚBLICA
}

\author{
Báltica Cabieses ${ }^{1,2, a}$, Piedad Gálvez²,b Nassim Ajraz ${ }^{2, c}$
}

\begin{abstract}
RESUMEN
La evidencia demuestra los altos niveles de vulnerabilidad que rodean al fenómeno migratorio en la actualidad. Dichas condiciones son de importancia para los sistemas de salud en todo el mundo, al vincularse directamente con el empeoramiento del estado de salud y calidad de vida de las personas. Sin embargo, el estudio del nexo entre el proceso migratorio y la salud se mantiene insuficiente, más aún respecto a cómo la migración internacional influye en la toma de decisión en salud pública. En la región Latinoamericana y del Caribe, existen iniciativas para prestar servicios de salud a migrantes internacionales, sin embargo, aún distan mucho de garantizar los derechos fundamentales en salud de quienes migran. Este artículo tiene como objetivo desarrollar una discusión crítica acerca de las principales teorías sociales sobre migración, y sobre su aporte a la toma de decisiones en salud pública (teoría de «tiraje y empuje», teoría de globalización, teoría de acumulación causal y el transnacionalismo). Se realizó una revisión narrativa académica hasta abril del 2017, cuyos resultados indican que es primordial la acción institucional, estatal y local de la salud pública para garantizar los derechos de salud de la población migrante frente al escenario de precariedad que rodea el fenómeno migratorio. Se espera que este documento sea un aporte al conocimiento actual en América Latina, tanto para la docencia como para la práctica e investigación en salud pública.
\end{abstract}

Palabras clave: Migración humana; Derechos de la salud pública; Vulnerabilidad en salud; América Latina; Caribe (fuente: DeCS BIREME).

\section{INTERNATIONAL MIGRATION AND HEALTH: THE CONTRIBUTION OF MIGRATION SOCIAL THEORIES TO PUBLIC HEALTH DECISIONS}

\begin{abstract}
Evidence demonstrates the high levels of vulnerability that surround the current migration phenomenon. These conditions are crucial to health systems worldwide, as they are directly linked to worsening health status and quality of life. However, the study of the connection between the migration process and health remains insufficient, especially with regard to how international migration influences public health decision-making. In the Latin American and Caribbean region, there are initiatives to provide health services to international migrants, but they are still far from guaranteeing the fundamental health rights of those who migrate. This article aims to develop a critical discussion of the main social theories on migration, and its contribution to public health decision-making (push-pull theory, globalization theory, causal accumulation theory, and transnationalism). An academic narrative review was carried out until April 2017, the results of which indicate that institutional, state, and local public health action is essential to guarantee the health rights of the migrant population in the face of the precarious situation surrounding the migration phenomenon. It is hoped that this document will be a contribution to current knowledge in Latin America, both for teaching and for practice and research in public health.
\end{abstract}

Keywords: Human migration; Health law; Health vulnerability; Latin america; Caribbean region (source: MeSH NLM).

\footnotetext{
Department of Health Sciences, University of York. York, England, UK

Facultad de Medicina Clínica Alemana, Universidad del Desarrollo. Santiago, Chile

Enfermera, matrona, PhD Health Sciences; ${ }^{\mathrm{b}}$ Socióloga; ${ }^{\mathrm{c}}$ Sociólogo

Recibido: 04/09/2017 Aprobado: 04/04/2018 En línea: 09/07/2018
}

Citar como: Cabieses B, Gálvez P, Ajraz N. Migración internacional y salud: el aporte de las teorías sociales migratorias a las decisiones en salud pública. Rev Peru Med Exp Salud Publica. 2018;35(2):285-91. doi: 10.17843/rpmesp.2018.352.3102 


\section{INTRODUCCIÓN}

La migración posee una naturaleza compleja y dinámica, como un proceso social sobre el cual operan determinantes económicos, políticos, socioculturales, individuales y tecnológicos. Las cifras del 2015 evidenciaron un aumento de $41 \%$ en la migración internacional con relación al $2000{ }^{(1)}$, guiando diversas disciplinas hacia el estudio de esta temática. Sin embargo, el énfasis en la relación entre el proceso migratorio y la salud se mantiene insuficiente, más aún en la temática de cómo la migración internacional influye en la toma de decisión en salud pública.

Amplia evidencia demuestra los altos niveles de vulnerabilidad que rodean al fenómeno migratorio en la actualidad (2), aspecto de suma relevancia para las instituciones sociales, políticas y económicas de los países que experimentan los efectos de la migración. Sin embargo, las condiciones de vulnerabilidad poseen especial importancia para los sistemas de salud en todo el mundo. El enfoque de determinantes sociales de la salud vislumbra el gran impacto que tienen estas vulnerabilidades en el empeoramiento del estado de salud y calidad de vida de las personas. En América Latina y el Caribe, preocupa la proporción desconocida de migrantes que residen hoy en situación irregular o en procesos de migración circular (en constante movimiento según oportunidades laborales) con niveles mínimos de protección y seguridad.

El propósito de este artículo es desarrollar una discusión crítica en el campo de estudio de la migración y la salud pública en Latinoamérica y el Caribe; el artículo muestra evidencia sobre las condiciones de salud implicadas en el proceso migratorio en la región; así como las principales teorías sociales sobre migración internacional, tanto clásicas como contemporáneas; permitiéndonos reflexionar sobre su aporte a la toma de decisiones en salud pública. Se espera que este documento sea un aporte al conocimiento actual en salud pública, tanto para docencia como para la práctica y la investigación.

\section{METODOLOGÍA}

Se realizó una revisión narrativa de la literatura académica, por medio de una búsqueda en Scopus, ProQuest, Scielo para fuentes en inglés y español publicadas hasta abril del 2017, usando la siguiente combinación de palabras claves: migración internacional, teorías migratorias, salud en población migrante, Latinoamérica y salud pública.

Se encontraron 3382 documentos que luego se filtraron según pertinencia y relevancia, utilizando como filtro las mismas palabras claves de la búsqueda, al corresponder a las áreas temáticas de interés. La selección de los artículos estuvo a cargo por todos los investigadores. Se seleccionaron
17 artículos científicos y 15 documentos de literatura gris donde se analizaron teorías sociales y de políticas de salud. Finalmente, se agregaron siete documentos luego de la revisión de expertos. Para el análisis, se utilizó un abordaje inductivo general, centrado en la temáticas de interés.

\section{ANÁLISIS DE LA SALUD DE PERSONAS MIGRANTES INTERNACIONALES DE LA REGIÓN LATINOAMERICANA Y DEL CARIBE}

Utilizando las cuatro fases del proceso migratorio como base analítica ${ }^{(3)}$ a) antes de la partida o abandono, b) tránsito o desplazamiento, c) inmigración o asentamiento en la comunidad de destino y d) retorno o reasentamiento, se expondrán las problemáticas sociales que operan en Latinoamérica y el Caribe y que desafían a los sistemas de salud. También, se darán a conocer algunas de las medidas tomadas en nuestra región.

\section{ANTES DE LA PARTIDA O ABANDONO}

Las condiciones socioeconómicas y políticas en el lugar de origen no sólo son algunos de los factores motivadores de la migración, sino que influencian directamente el estado de salud previo al viaje ${ }^{(4,5)}$. Dentro de los factores de riesgo para la salud en la primera fase del proceso encontramos la pobreza, la marginalización de servicios sociales básicos (como la educación y salud) y los conflictos políticos. En la realidad del continente resulta de especial preocupación aquellas situaciones que derivan de corrientes migratorias forzosas, como la violación de derechos humanos, la violencia sexual o la guerra ${ }^{(3)}$.

En América Latina y el Caribe, uno de los principales desencadenantes de la migración forzada corresponde a la proliferación del crimen organizado y la violencia de género sobre todo en países centroamericanos, tal como constató Willers et al. (6). Asimismo, en su estudio sobre la salud mental de migrantes en situación de irregularidad, TemoresAlcántara ${ }^{(7)}$ identificó que aquellos que sufrieron migración forzada experimentaron daños en su salud mental, producto de la persecución en sus propios países, junto a la falta de preparación para dejar su hogar.

Los países emisores enfrentan múltiples desafíos para salvaguardar la salud de su población, siendo el desarrollo de estrategias contra la trata y tráfico de personas, uno de los prioritarios. Un ejemplo de acciones concretas es Colombia, donde la coordinación entre la política nacional y las organizaciones internacionales, logró disminuir delitos y detectar redes de tráfico de personas ${ }^{(8)}$.

\section{TRÁNSITO O DESPLAZAMIENTO}

Duranteel desplazamiento, los migrantesen situación irregular, refugiados y desplazados se ven especialmente expuestos a situaciones que pueden afectar su salud ${ }^{(3,9)}$. La principal ruta de migrantes en situación irregular en Latinoamérica 
corresponde al corredor migratorio Centroamérica-MéxicoEstados Unidos, actualmente uno de los trayectos más inseguros y violentos del mundo ${ }^{(10)}$. Esta situación se ha agudizado por el incremento de políticas restriccioncitas en México y Estados Unidos, promoviendo el uso de rutas de mayor riesgo para eludir controles migratorios ${ }^{(10,11)}$. En dichas rutas quienes migran son susceptibles de ser víctimas de violencia física o sexual en manos de organizaciones criminales que controlan el tráfico de drogas y de migrantes en la región ${ }^{(6)}$. Diversos estudios han documentado altos niveles de estrés, angustia y ansiedad en quienes han transitado de manera irregular por México (7). Por otro lado, se suelen presentar condiciones precarias durante el viaje; sin acceso a servicios básicos como agua potable, alimentación o alojamiento; lo que ocasiona cuadros de deshidratación, hipotermia, asfixia, estrés y ansiedad ${ }^{\left({ }^{9}\right.}$.

Ante este escenario adverso de falta de atención médica y de servicios básicos ${ }^{(12)}$ surgen iniciativas como la implementación en México de la afiliación temporal al Seguro Popular para migrantes en tránsito, lo que permite el acceso a prestaciones del sistema social en salud sin tener que presentar documentos ${ }^{(13)}$. En el Estado de Tabasco (México) también se realizan esfuerzos para la atención en salud de migrantes, sin embargo, su alcance se ve limitado por aspectos como la difusión de información de la oferta de servicios ${ }^{(14)}$.

\section{ASENTAMIENTO EN LA SOCIEDAD DE DESTINO}

Respecto a la etapa de asentamiento en el país de destino, existe amplia evidencia de que el fenómeno de marginalización social y cultural que enfrentan algunos grupos conlleva a una serie de problemáticas en salud (6). Por otro lado, el acceso a servicios de salud es diferente en cada país. Ciertos países receptores que han desarrollado normativas para garantizar el acceso a salud en igualdad a la población son Argentina, Brasil y Uruguay ${ }^{(13)}$, mientras que en países como Estados Unidos se mantiene una gran brecha de derechos sociales entre migrantes y nacionales, que también afecta la salud ${ }^{(15)}$. Este escenario perjudica especialmente a los migrantes en situación de irregularidad, ya que suelen evadir los mecanismos formales de atención por el miedo a ser deportados, recurriendo a la automedicación, al envío de medicamentos desde el país de origen, o al traslado a ciudades mexicanas en búsqueda de atención médica ${ }^{(16)}$.

Respecto a la salud mental, la migración implica una serie de eventos estresantes como la adaptación a un nuevo país, la discriminación y la lejanía de los seres queridos, problemática conocida como estrés aculturativo (16). Evidencia recolectada en Estados Unidos indica que la problemática llega a afectar a muchos migrantes latinos debido a sentimientos de rechazo, generando conflictos de identidad, enfermedades psicosomáticas y/o depresión ${ }^{(6)}$.

Es importante destacar el rol que cumplen diversas organizaciones sociales no estatales (ONG) en los países de destino, al prestar apoyo a migrantes en situación de irregularidad en vivienda y atención médica. Sin embargo, se ha demostrado que estas iniciativas no reemplazan una política o ley enfocada la disminución de la brecha de los derechos (entre ellos de salud) de los migrantes, pues no responden sistemáticamente a las necesidades emergentes llevando a cuestionar su eficiencia en vista de los desafíos actuales ${ }^{(15)}$.

\section{RETORNO O MOVILIDAD A OTRO LUGAR}

Las cifras indican un aumento de la población en retorno, a nivel internacional, en la última década ${ }^{(17)}$, sin embargo, la heterogeneidad de sus causas junto al aumento del fenómeno de migración circular hace difícil su medición y teorización ${ }^{(18)}$. En su investigación sobre migrantes peruanos retornados desde Italia, Nieto ${ }^{(18)}$ observó que, quienes retornan no necesariamente incrementan su capital humano durante la migración (como sostiene la literatura), sino que, factores como haber descendido en el escalafón social en el país de destino y el desgaste físico junto al avance en el ciclo de vida, influyen en la pérdida relativa e incluso absoluta de su capital humano. Por su parte, Cerrutti y Maguid (17) indagaron en comunidades sudamericanas en retorno desde España, concluyendo que los inmigrantes más vulnerables en términos laborales, documentarios y con menor antigüedad migratoria, fueron los más proclives a volver al origen tras los embates de la crisis económica.

Un caso especial corresponde a las personas que sufren la deportación ${ }^{(3)}$, quienes relatan episodios de violencia física y psicológica, incluso robo y extorsión, produciendo traumas que los servicios públicos de los países de origen deben ser capaces de abordar ${ }^{(12)}$. En este nivel resultan útiles las estrategias de reintegración, que garanticen el acceso a servicios de salud con un particular enfoque psicológico. Un ejemplo es el Programa Integral de Inserción en EI Salvador, o la atención personalizada ofrecida en México a través la estrategia "Somos Mexicanos» ${ }^{(19)}$, que orienta y vincula a redes de apoyo a personas retornadas.

\section{TEORÍAS SOCIALES MIGRATORIAS Y SU APORTE A LA TOMA DE DECISIÓN EN SALUD PÚBLICA}

\section{TEORÍA DEMOGRÁFICA CLÁSICA DE «TIRAJE Y EMPUJE»}

Ravenstein en 1885 fue el primero en usar de forma implícita el modelo analítico de «atracción y repulsión». Dicho modelo, basado en el paradigma de la época ${ }^{(20)}$, concebía al humano como un ser libre y racional, con capacidad de elegir entre posibilidades que maximizan sus beneficios y minimizan sus costos. Por consiguiente, Ravenstein planteó que el mayor volumen de los flujos migratorios se explica por el deseo individual de obtener mejores condiciones de vida material, desplazándose 
desde lugares excedentes de población hacia destinos con alta demanda de mano de obra, producto del desarrollo industrial y comercial ${ }^{21)}$. Lee en 1966 fue quien explicitó el modelo de Ravenstein, postulando factores de «tiraje» o atracción y otros de «empuje» o alejamiento, siendo factores de «empuje» a emigrar los bajos salarios, malas condiciones de empleo o limitadas oportunidades de educación o desarrollo, mientras que son factores de «tiraje» fueron mejores salarios o condiciones de trabajo, sistemas universales de protección social y salud, entre otros ${ }^{(22)}$.

Las principales críticas a esta teoría se relacionan con la simplificación de la migración a factores puramente demográficos y económicos, cuestionando el hecho de que diferencias poblacionales de escala internacional afecten las decisiones individuales. Por otro lado, se cuestiona que la migración no se explica a cabalidad por el funcionamiento del mercado del trabajo y las diferencias de ingresos esperadas ${ }^{(23)}$. Se debe tener presente el enfoque propio del contexto en que nace la teoría, donde se omitía el entorno social y político que puede coartar la acción de los sujetos en el desarrollo de procesos migratorios ${ }^{(21)}$.

\section{TEORÍA MIGRATORIA DESDE «LA GLOBALIZACIÓN»}

Los orígenes de la globalización suelen remitirse a la nueva configuración mundial que tomó lugar en la segunda mitad del siglo $X X$, a partir de la consolidación del neoliberalismo a nivel mundial ${ }^{24)}$. Desde 1970, la rápida adherencia de los Estados a este modelo político-económico significó una profunda transformación de las relaciones entre países y regiones, debido al desarrollo de políticas de libertad empresarial y marcos institucionales de propiedad privada ${ }^{(24)}$. El fenómeno de globalización se encuentra estrechamente vinculado a la unificación del espacio mundial ${ }^{25)}$, en la libre circulación de bienes y servicios, en la creación de un mercado globalizado, y en la apertura de las comunicaciones, la información y los transportes.

«La era de la migración», concepto introducido por Stephen Castles y Mark J. Miller en 1993, teoriza los efectos de la globalización sobre la migración internacional, constatando una aceleración, diferenciación y politización de los flujos migratorios ${ }^{(11)}$. Así también Arango ${ }^{(25)}$ afirma que la migración del mundo globalizado se caracteriza principalmente por la diversificación de los flujos en un doble sentido, la mayoría de los países del planeta participan en la migración internacional, y las personas van de una parte a cualquier otra, multiplicándose las rutas migratorias. En el caso latinoamericano, Marroni (11) explica que, si bien los desplazamientos hacia Estados Unidos poseen gran relevancia en el continente, en nuestro mapa migratorio se presentan al menos cinco dinámicas relevantes, donde se encuentran migraciones fronterizas y migraciones regionales o extra-regionales no fronterizas intercontinentales.
Otra apreciación destacable corresponde a que la eliminación de barreras internacionales para la libertad productiva y comercial no vino acompañada de la supresión de barreras para la movilidad de la fuerza de trabajo (11), sino más bien de doctrinas de seguridad nacional en gran parte de los países del mundo. Se manifestaría así una gran contradicción entre la unificación comercial a nivel mundial y la restricción y control de la movilidad humana ${ }^{(25)}$. Por lo tanto, se concluye que las dinámicas migratorias de la globalización, diversas y múltiples, ocurren a pesar de las políticas de restricción, y no gracias a su disminución.

\section{TEORÍA MIGRATORIA DE «ACUMULACIÓN CAUSAL»}

Esteenfoquebusca explicarporquélamigración internacional se perpetúa en el tiempo una vez iniciada ${ }^{(21,26)}$. La «causalidad acumulativa» fue un proceso identificado por Gunnar Myrdal en 1957 y desarrollado por Douglas Massey en 1990 en el estudio de la inmigración mexicana en Estados Unidos (26). Sostiene que, durante el proceso migratorio, surgen nuevas condiciones que operan como causas en sí mismas para el mantenimiento de los flujos ${ }^{(27)}$; estas corresponderían al fenómeno de «cadena de la migración» ${ }^{(28,29)}$, donde cada acto de migración altera el contexto social de las sociedades que participan, facilitando los movimientos migratorios a través del tiempo. Cada nueva migración reduciría el costo de futuras migraciones, creando un cambio de valores y de cultura, que a su vez facilitarán futuras migraciones ${ }^{(28)}$. Las redes sociales serían útiles para la decisión de migrar; en muchos casos, la comunidad migrante compartiría además relaciones familiares y de amistad ${ }^{(28)}$. Postula que la cadena de migraciones tendría el potencial de influir positivamente en los países de origen, ya sea por medio de remesas, la búsqueda de buenas relaciones políticas internacionales o acuerdos de largo aliento.

Las críticas que ha recibido esta teoría es que no desarrolla en profundidad la «migración circular» de personas que no se detienen en un solo país, como tampoco profundiza en las consecuencias sociales y políticas del retorno de los migrantes a sus países de origen ${ }^{(28)}$.

\section{EL TRANSNACIONALISMO}

El transnacionalismo es considerada una de las teorías más importantes en la temática migratoria, desarrollada por Glick Schiller, Basch \& Blanc-Szanton en 1992 y 1994 (30). Bajo la necesidad de abandonar la tradicional figura del «inmigrante» es que surge el concepto de «transmigrante», y refiere a que las personas que migran construyen espacios sociales en donde conectan, de manera simultánea, sus países de origen y de destino, en un proceso continuo ${ }^{(31)}$. A diferencia de los estudios clásicos enfocados en las causas para emigrar o en la adaptación en el destino, el transnacionalismo se interesa en las relaciones que son mantenidas entre ambos lugares, y en el movimiento de personas, bienes, información y material simbólico que traspasan las fronteras ${ }^{(32)}$. 
Mientras que la propuesta de Glick Schiller, Basch \& Blanc-Szanton incorpora todo el espectro de relaciones, diferenciándolas entre aquellas más institucionalizadas (como las de tipo económicas o políticas) de aquellas menos institucionalizadas (culturales o familiares) (30), autores como Portes en 1994 dan mayor relevancia a las relaciones transnacionales de tipo económicas, tales como las remesas monetarias, los empresarios migrantes y las remesas colectivas ${ }^{(30)}$. Por último, autores como Mitchell desarrollan un transnacionalismo geográfico ${ }^{(33)}$, donde el movimiento en sí, junto a los agentes que intervienen su desarrollo, toman preponderancia.

Los aspectos más valorados de esta teoría corresponden a su estrecho vínculo con el estudio empírico ${ }^{(33)}$, junto a su carácter novedoso al permitir el análisis de los efectos de la migración entre sociedades de origen y de destino. También posibilita integrar factores micro, meso y macro estructurales que operan en las migraciones ${ }^{(33)}$.

\section{DISCUSIÓN PARA LATOMA DE DECISIÓN EN SALUD PÚBLICA}

Dentro de las cuatro teorías abordadas, unas favorecen nuestra comprensión sobre elementos que, a un macro nivel, influyen sobre la calidad de vida y el estado de salud de los migrantes, mientras que otras poseen mayor poder explicativo a niveles meso y micro.

A un nivel macro estructural, la teoría de «tiraje y empuje» nos permite reconocer la ventaja demográfica y de fuerza de trabajo que los grupos migrantes ofrecen, sobre todo a países envejecidos y con rápida reducción de su fuerza laboral. Ello indica el deber de dichos países de planificar y proyectar sus capacidades, sus recursos materiales y humanos, para brindar atención de calidad a esta población. Sin embargo, dado que el deterioro de la salud puede ser enfrentado en gran medida en las sociedades de origen, es también necesario que estas pongan en práctica políticas en salud que integren y apoyen de manera específica a los migrantes y sus familias.

En la región Latinoamericana y del Caribe, ciertos países receptores cuentan con iniciativas a nivel de leyes o políticas que buscan otorgar prestaciones en salud a migrantes internacionales. Si bien no corresponden a políticas de salud migratorias, estas logran administrar la coordinación intersectorial, la capacitación de trabajadores de la salud y las estrategias para ampliar la cobertura y acceso a servicios ${ }^{(13)}$. Por su parte, la teoría desde la globalización incorpora una perspectiva de desigualdad socioeconómica y contradicción a escala global; brechas económicas entre países que se posicionan en un mercado mundial se definen como motor de los movimientos humanos, siendo las doctrinas de seguridad nacional o de restricción aquellas causantes de la precariedad que afecta a los flujos provenientes de países con menor capital económico.
A un nivel meso o intermedio, el transnacionalismo nos muestra la presencia cada vez mayor de espacios transnacionales en Latinoamérica y el mundo, donde flujos multidireccionales de personas, grupos familiares, productos y empresas, traspasan las fronteras ${ }^{(32)}$. Fenómenos como el crimen trasnacional y el tráfico de personas, demandan medidas que operen a niveles internacionales.

Para la salud pública es de suma relevancia proyectarse hacia una población multicultural y en movimiento, haciendo necesario avanzar hacia una mayor colaboración y comunicación entre naciones que experimentan simultáneamente los efectos de la migración. Por su parte, la teoría de «acumulación causal» permite reconocer actores claves para abordar la colaboración internacional. Es fundamental el papel que juega el apoyo social que pueden brindar las redes migratorias, capital social que se puede transferir en distintas formas: información necesaria, apoyo material y emocional. En este nivel destaca como iniciativa dentro de la región la Organización del Frente Indígena Mixteco activa en Estados Unidos y México ${ }^{(33)}$ que funciona como una red de solidaridad para los mexicanos que pasan la frontera. Las ONG, casas de acogida y albergues también socorren las múltiples vulnerabilidades que presentan los migrantes, las redes formales e informales de migrantes ayudan a sobrellevar los desafíos del proceso, señalando la importancia de la cohesión y capital social como mediadores de la migración internacional y su sostenimiento en el tiempo.

A un micro nivel, vimos cómo la sensación de desadaptación sociocultural, el temor a la deportación, la discriminación, la violencia y la violación de derechos, son problemáticas presentes en varias de las fases de la migración latinoamericana. La salud pública necesita enfrentarse al vínculo entre la migración -como un todo complejo- y la proliferación de problemas de salud mental como la ansiedad, el estrés e incluso la depresión, requiriendo sistemas de salud capaces de apoyar psicosocialmente a quienes lo necesitan.

A partir de la evidencia revisada ${ }^{(13)}$, se propone que dentro las prácticas y acciones implementadas desde la salud pública, se presente de manera explícita el concepto de derechos humanos como su principio rector y fundamento de acciones concretas en materia de salud. El concepto de derechos humanos corresponde a uno de los fundamentos esenciales para la igualdad en salud entre los grupos nacionales y extranjeros. Para esto se debe reconocer de forma explícita el derecho a la salud de los migrantes en los territorios nacionales, lo que requiere de un instrumento normativo específico que se refiera al derecho a la salud de los migrantes, idealmente en la misma política migratoria del país. 


\section{REFLEXIONES FINALES}

Una de las principales ventajas que supone el estudio teórico-empírico del fenómeno migratorio, tal como argumenta este trabajo, es el poder mostrar factores macro o de escala global, meso o de escala sociocultural y micro o de escala familiar-individual, que inciden de manera importante sobre el modo en que acontece el fenómeno migratorio en el presente. Ello indica comprender el rol de la salud pública en un mundo globalizado, los niveles mínimos de protección y seguridad subyacentes a la restricción de la movilidad humana, los efectos sociales y de salud presentes en países emisores y receptores de la migración, la importancia de las redes y el capital social para quienes migran en el acceso a servicios y prestaciones básicas como la salud, entre otros.

Una de las principales medidas o políticas para enfrentar esta problemática corresponde a la incorporación institucional de la población migrante, como respuesta a la garantización de los derechos humanos y salud de las personas migrantes internacionales. Si bien dentro de los países Latinoamericanos y del Caribe existen iniciativas para la integración de personas migrantes internacionales a la salud pública, todavía en ninguno se ha desarrollado una política de salud migratoria propiamente ${ }^{(13)}$. Se vuelve explícita la necesidad de que en todo el continente se plantee con urgencia el asegurar el derecho a salud de todas las personas. En la región también son escasos los acuerdos binacionales o internacionales de salud (hasta la fecha existen sólo cuatro) ${ }^{(13)}$, lo que dificulta la planificación y cooperación entre países que poseen espacios sociales cada vez más multiculturales, transnacionales y dinámicos. Ciertamente, lo que no se puede hacer es negar el fenómeno migratorio que la mayoría de los países enfrenta en la actualidad. Se requiere de una voz clara desde la salud pública para recalcar el valor de la migración internacional, y buscar formas de protección de la vida y salud de las poblaciones. En este sentido, este ensayo sintetiza y discute el aporte a la salud pública de las cuatro principales teorías sociales sobre migración internacional, tanto clásicas como contemporáneas. Adicionalmente, aporta a la relación entre migración internacional y salud poblacional, al incorporar aproximaciones teórico-empíricas de gran alcance en la actualidad: la determinación social de la salud (35), la desigualdad social en salud internacional y local (35), el enfoque de salud en todas las políticas ${ }^{(36)}$, y la traslación del conocimiento de investigación a la acción en salud pública ${ }^{(37)}$. Se espera que este documento sea un aporte al conocimiento actual en la región de Latinoamérica, tanto para docencia como para la práctica y la investigación.

\section{LIMITACIONES DEL ESTUDIO}

Al centrar el análisis del fenómeno de vulnerabilidad en el proceso migratorio en Latinoamérica y el Caribe, no se abordó toda la complejidad del fenómeno, sugiriendo que investigaciones en el futuro aborden las temáticas vistas por este ensayo en otros contextos y a diferentes niveles. Por otro lado, al realizar una selección de información no sistemática y al incluir fuentes exclusivamente en español e inglés, se dejó fuera mucha información relevante para el estudio que pudo haber contribuido a nuestros propósitos, como es el caso de la información que se encuentra en portugués.

Contribuciones de autoría: BC, PG y NA participaron en la concepción del artículo, la recolección y análisis de datos, su redacción y aprobación de la versión final. BC obtuvo el financiamiento.

Financiamiento: El presente artículo obtuvo su financiamiento a través del proyecto FONDECYT 11130042: «Desarrollando inteligencia en salud pública primaria para migrantes internacionales en Chile: un estudio multi-métodos (20132017)», y «Proyecto Interfacultades UDD 2016», realizado por el Programa de Estudios Sociales en Salud de la Facultad de Medicina de la Universidad del Desarrollo».

Conflicto de interés: No.

\section{REFERENCIAS BIBLIOGRÁFICAS}

1. United Nations. Trends in international migration, 2015 [Internet]. New York: Department of Economic and Social Affairs, UN; 2015.

2. Fuertes C, Martín Laso MA. El inmigrante en la consulta de atención primaria. Anales Sis San Navarra. 2006;29(1):9-25.

3. Organización Internacional para las $\mathrm{Mi}$ graciones (OIM). Migración Internacional, Salud y Derechos Humanos [Internet]. Ginebra: OIM; 2013. Disponible en: http://publications.iom.int/system/files/ pdf/iom_unhchr_sp_0.pdf

4. Cabieses B, Bernales M, McIntyre A. La migración internacional como determinante social de la salud: evidencia y pro- puestas para políticas públicas [Internet]. Santiago: Universidad del Desarrollo; 2017 enero 2017. 529 p. Disponible en: http://www.udd.cl/dircom/pdfs/Libro_ La_migracion_internacional.pdf

5. Organización Internacional para las Migraciones (OIM). Manual de Referencia, Migración Saludable en América Central [Internet]. Ginebra: OIM; 2012. Disponible en: http://publications.iom.int/system/ files/pdf/manual_referencia_1.pdf

6. Willers S. Migración y violencia: las experiencias de mujeres migrantes centroamericanas en tránsito por México. Sociologica (Méx). 2016;31(89):163-95.
7. Temores-Alcántara G, Infante C, Caballero M, Flores-Palacios F, Santillanes-Allande N. Salud mental de migrantes centroamericanos indocumentados en tránsito por la frontera sur de México. Salud Publica Mex. 2015;57(3):227-33.

8. Ripoll De Castro A. Política preventiva frente a la trata de personas seguridad versus derechos humanos Rev Relac Int Estrateg Segur. 2010;5(2):235-49.

9. Organización Internacional para las Migraciones (OIM). Lineamientos estratégicos para el abordaje de las personas migrantes en tránsito por Mesoamérica [Internet]. San Jose: Oficina Regional para 
Centroamérica, Norteamérica y el Caribe, OIM; 2017. Disponible en: http:// saludymigracion.org/es/system/files/ repositorio/lineamientos_estrategios_ para_el_abordaje_de_la_salud_de_las_ personas_migrantes_en_transito_por_ mesoamerica_2017_0.pdf

10. López Recinos V. Desarrollo, migración y seguridad: El caso de la migración hondureña hacia Estados Unidos. Migr Desarro. 2013, 11(21):65-105.

11. Marroni M. Escenarios migratorios y globalización en América Latina: una mirada al inicio del siglo XXI. Pap Trab Cent Estud Interdiscip Etnolingüíst Antropol Soc. 2016;32.

12. Organización Internacional para las Migraciones (OIM). Condición de salud, acceso a los servicios e identificación de riesgos y vulnerabilidades asociados a la migración en El Salvador [Internet]. San José: Oficina Regional para Centroamérica, Norteamérica y el Caribe, OIM; 2015. Disponible en: http:// saludymigracion.org/es/system/files/ repositorio/condicion_de_salud_el_ salvador.pdf

13. Cabieses B; Alarcón A; Bernales M; Astorga S, Martinez F. Informe Final Estudio Licitación Sistematización y Evaluación del Piloto Nacional de Salud de Inmigrantes. Santiago: Ministerio de Salud de Chile. En colaboración con Organización Internacional para las Migraciones y Universidad del Desarrollo; 2018

14. Santos Padrón H. Limón Mercado N. Martínez Calvo S. La atención de los migrantes Centroamericanos en los servicios de Salud en Tabasco, 2012-2014. Horiz sanitario. 2016;15(3):143-53.

15. Frey B, Pardo AM. Filling the Migrant Rights Gap: Localized Protections of International Economic and Social Rights. Public Integrity. 2017(19):136-50.

16. Nigenda G, Ruiz-Larios JA, BejaranoArias RM, Alcalde-Rabanal JE, BonillaFernández P. Análisis de las alternativas de los migrantes mexicanos en Estados Unidos de América para atender sus problemas de salud. Salud Publica Méx. 2009;51(5):407-16.

17. Cerrutti MA, Maguid A. Crisis económica en España y el retorno de inmigrantes sudamericanos. Migr Inter. 2016;8(3):155-89.
18. Nieto C. Motivaciones de la migración de retorno. ¿Qué implicaciones para el desarrollo? Crisis Global y Estrategias Migratorias: hacia la redefinición de las políticas de movilidad. En: IV Congreso de la Red Internacional de Migración y Desarrollo. 18,19 y 20 de mayo de 2011. Quito: FLACSO; 2011. Disponible en: http://www. flacsoandes.edu.ec/system/tdf/agora/ files/1308692461.ponencia_carlos_nieto. pdf ?file $=1 \&$ type $=$ node\&id $=62015$

19. Comisión Económica para América Latina y el Caribe (CEPAL). Informe de la reunión extraordinaria de la mesa directiva de la conferencia regional sobre población y desarrollo de América Latina y El Caribe [Internet]. Santiago: CEPAL; 2017. Disponible en: https://repositorio.cepal. org/bitstream/handle/11362/43289/1/ S1800134_es.pdf

20. Blanco C. Las migraciones contemporáneas. Madrid: Alianza Editorial; 2000.

21. Vila-Belda JA. Las «Leyes de las Migraciones» de E. G. Ravenstein, cien años después. Reis: Rev Esp Investig Sociol. 1985;32(85):7-26.

22. Mager Hois EA. Migración transfronteriza de los Kikapú y sus efectos de identidad Multidisciplina. 2014;18:148-77.

23. García Abad R. Un estado de la cuestión de las teorías de las migraciones. Historia Contemporánea 2003;26:329-51.

24. Harvey D. Breve historia del Neoliberalismo. Madrid: Akal; 2007.

25. Arango J. Las Migraciones Internacionales en un Mundo Globalizado. Madrid: Universidad Complutense de Madrid; 2007.

26. Massey DS, Durand, J, Malone, N. Detrás de la trama. Políticas migratorias entre México y Estados Unidos. Zacatecas: Universidad Autonoma de Zacatecas; 2009.

27. Massey DS, Arango J, Graeme H, Kouaouci, A, Pellegrino A, Taylor E. Teoría sobre la migración internacional: una reseña y una evaluación. 2000; Trabajo2(3).

28. Martine G, Hakkert R, Guzmán JM. Social aspects of international migration: Preliminary Considerations International migration and development in the Americas [Internet]. En: International migration and development in the Americas. San Jose de Costa Rica: Economic Commission for Latin America and the Caribbean (ECLAC); 2000. Disponible en: https://repositorio.cepal. org/bitstream/handle/11362/6533/ S017586_en.pdf

29. Greenwood MJ. Human migration: theory, models, and empirical studies Journal Reg Sci. 1985;25(4):521-44.

30. Pardo AM. Migración internacional y desarrollo. Aportes desde el transnacionalismo Rev Estud Soc. 2015;52:39-51.

31. Schiller NG, Basch L, Blanc-Szanton C. Transnationalism: A New Analytic Framework for Understanding Migration. Ann N Y Acad Sci. 1992;645:1-24.

32. Ciurlo A. Género y familia transnacional. Un enfoque teórico para aproximarse a los estudios migratorios. Rev Cient Gen José María Córdova 2014;12(13):127-61.

33. Vilar E, Eibenschutz C. Migración y salud mental: un problema emergente de salud pública. Rev Gerenc Polit Salud 2007;6(13):11-32.

34. Vertovec S. Transnacionalism. Londres: Routledge; 2009.

35. Irwin A, Valentine $\mathrm{N}$, Brown $\mathrm{C}$, Loewenson R, Solar O, Brown H, et al. The commission on social determinants of health: tackling the social roots of health inequities. PLoS Med. 2006;3(6):e106.

36. Yach D. 'Health-for-All' in the twentyfirst century: a global perspective. Natl Med J India. 1997;10(2):82-9.

37. Cabieses B, Espinoza MA. La investigación traslacional y su aporte para la toma de decisiones en políticas de salud. Rev Peru Med Exp Salud Publica. 2011;28(2):288-97.

\section{Correspondencia: Baltica Cabieses.}

Dirección: Facultad de Medicina Clínica Alemana, UniversidaddelDesarrollo, Santiago, Chile. Avenida La Plaza 680 San Carlos de Apoquindo Las Condes, Santiago, Chile. Correoelectrónico:bcabieses@udd.cl 